\title{
Overview of emerging treatment of non-alcoholic fatty liver disease: more than one drug needed?
}

\author{
Dong Yun Kim ${ }^{1,2}$, Jun Yong Park ${ }^{2,3,4}$ \\ ${ }^{1}$ Department of Pharmacology, Yonsei University Graduate School of Medicine, Seoul, South Korea; ${ }^{2}$ Department of Internal Medicine, ${ }^{3}$ Institute of \\ Gastroenterology, Yonsei University College of Medicine, Seoul, South Korea; ${ }^{4}$ Yonsei Liver Center, Severance Hospital, Seoul, South Korea \\ Correspondence to: Jun Yong Park, MD, PhD. Department of Internal Medicine, Yonsei University College of Medicine, 50-1 Yonsei-ro, Seodaemun- \\ gu, Seoul 03722, South Korea. Email: drpjy@yuhs.ac. \\ Comment on: Friedman SL, Neuschwander-Tetri BA, Rinella M, et al. Mechanisms of NAFLD development and therapeutic strategies. Nat Med \\ 2018;24:908-22.
}

Submitted Apr 01, 2019. Accepted for publication May 16, 2019.

doi: 10.21037/hbsn.2019.05.08

View this article at: http://dx.doi.org/10.21037/hbsn.2019.05.08

Non-alcoholic fatty liver disease (NAFLD) comprises isolated hepatic steatosis, non-alcoholic steatohepatitis (NASH), liver failure-associated complications of liver cirrhosis, and liver cancer. These are forecasted to be the leading liver diseases in the future. Identifying patients with NAFLD at highest risk for developing clinically meaningful outcomes is a key issue for managing therapy in those with NAFLD. Because fibrosis stage is a marker for determining clinical outcomes (1), it should be considered the most important treatment parameter. Generally, NASH patients without fibrosis are regarded to have a low risk of NAFLD-associated complications (2). NASH patients with no fibrosis (F0) or negligible fibrosis (F1) has an excellent prognosis; close follow-up and treatments are not requisite. However, the risks of liver-associated illness and death increase dramatically once bridging fibrosis (F3) or cirrhosis (F4) occurs in NAFLD, justifying much closer follow-up and monitoring to determine the longterm overall mortality from liver disease in these patients compared with those with little or no fibrosis $(3,4)$. Thus, it is crucial for clinicians to identify patients who have progressed to advanced NAFLD and classify them by severity of liver disease. For diagnosing and determining the severity of NAFLD and evaluating fibrosis, liver biopsy still remains the gold standard. However, there are many shortcomings in liver biopsy, including subjectivity of observer interpretation, high cost, and sample error. Because of these, many non-invasive methods using scoring systems, imaging tools, and various serum biomarkers have been tried as substitutes for liver biopsy and to subdivide NAFLD patients into different prognosis categories. For example, magnetic resonance elastography and Fibroscan ${ }^{\circledR}$ (Echosens, France), which are elastography-based methods, can be considered, although these have not yet been approved for this indication $(5,6)$. Even though optimal non-invasive diagnostic tools are still debated, clinicians should keep the importance of selecting high-risk patients in mind.

The primary approaches for NAFLD treatment are control of comorbid metabolic illnesses and lifestyle modifications, which are important for all patients. Also, a stage-based treatment approach should be considered. Most patients do not need pharmacological treatment, given the low likelihood of adverse liver-related outcomes in low-risk NAFLD patients. However, management is tailored according to the severity of fibrosis for patients who have more significant fibrosis (F2 or higher) when NASH is diagnosed. Nevertheless, the actual options for NASH treatment are restricted due to high individual variability and the rather complex nature of the disease. In a recent issue, Friedman et al. widely reviewed the potential candidate-drugs under evaluation in pre-clinical studies as well as those currently used to treat NAFLD in clinical practice (7). Unfortunately, none of the approachesmetabolic targeted therapies, anti-apoptotic agents, antiinflammatory or anti-fibrotic targeted therapies, and extrahepatic targeted therapies-have provided clear results even though many clinical trials are underway. The 
key challenge for clinicians that are waiting for new and effective therapy approvals is to understand the spectrum of liver disease in the context of disease progression. Several factors that promote inflammation, for example, visceral adiposity, changes in intestinal microbiota, and metabolic syndrome, increase hepatocyte exposure to cytokines, gutderived products, and other hepatotoxic inflammatory mediators. Several molecular pathways contribute to the development of NAFLD, and the mechanisms that lead to disease and clinical manifestations are highly heterogeneous. In clinical trials, dozens of medications are currently being tested to address these mechanisms (8). The therapies are diverse in their mechanisms of action which are currently focused on anti-inflammatory effects, anti-fibrotic effects, or metabolic targets. Among them, this editorial focuses on four medications that are emerging as pharmacological options in phase III clinical trials: cenicriviroc (CVC), elafibranor, selonsertib (SEL), and obeticholic acid (OCA).

CVC, a dual CCR2/5 (chemokine receptor 2 and 5) antagonist, demonstrated anti-fibrotic effects in preclinical models. NASH patients with fibrosis were treated with CVC $(150 \mathrm{mg} /$ day or placebo) for 24 months and histological improvement was evaluated in the phase IIb clinical trial. Analysis of a secondary endpoint, achieving a $\geq 1$-stage improvement in fibrosis, indicated that a greater number of patients in the CVC group (20\%) achieved an improvement in fibrosis compared with the placebo group (10\%) ( $\mathrm{P}$ value $=0.02)$, even though the difference in histological improvement was not meaningful between the placebo group (19\%) and the CVC groups (16\%), respectively ( $\mathrm{P}$ value $=0.52)(9)$. A phase III RCT is currently underway to see the effects of CVC $(150 \mathrm{mg} /$ day or placebo) on NAFLD patients with liver fibrosis (AURORA). The second category, elafibranor is a peroxisome proliferatoractivated receptor alpha/delta $(\mathrm{PPAR}-\alpha / \beta)$ agonist. Daily administration of the dual PPAR $\alpha / \beta$ agonist elafibranor $(80 \mathrm{mg})$ in obese subjects is associated with improved peripheral and hepatic insulin resistance (10). A recent randomized clinical trial showed that a daily oral dose of $120 \mathrm{mg}$ elafibranor for 52 weeks was associated with improvements in hepatic steatosis and fibrosis (11). Currently, the phase III RCT is underway to rate the effects of elafibranor $(120 \mathrm{mg} /$ day $)$ on histological improvement, liver-related complications, drug-related mortality, and cardiovascular disease at 72 weeks.

Third, SEL (GS-4997) is a selective apoptosis signalregulating kinase 1 (ASK1) inhibitor. To assess the effects of SEL (18, $6 \mathrm{mg} /$ day, placebo), two phase III studies have been started for NASH patients with compensated cirrhosis (STELLAR-4) or bridging fibrosis (STELLAR-3) to assess histological improvement with a planned analysis at 48 weeks. Unfortunately, both the STELLAR-3 and STELLAR-4 study failed to show a significant benefit in patients receiving the drug compared with the placebo group in the outcome of the week 48 analysis. Although these results are disappointing, additional sub-analyses of patients who responded optimally in the STELLAR program can be considered. Farnesoid X receptors (FXRs), are nuclear receptor transcription factors expressed in the liver that play a role in lipid metabolism. Interestingly, loss of FXR expression in mice amplified the ability of endoplasmic reticulum stress to drive expression of NACHT, LRR, PYD domain-containing protein 3 (NLRP3), and thioredoxin interacting protein, thereby augmenting liver injury. However, the FXR ligand had the opposite effect. FXR activation reduced the expression of NLRP3 by inhibiting the endoplasmic reticulum stressmediated PERK/CHOP pathway. These results indicate that FXR regulation of endoplasmic reticulum stress helps inhibit the liver injury associated with activation of the inflammasome (12). A phase III clinical trial is currently enrolling biopsy-proven NASH patients to see the effect of OCA $(25,10 \mathrm{mg} /$ day, placebo) on liver histology for 72 weeks. To assess long-term outcomes including progression to cirrhosis, death, or need for liver transplantation, those patients will be followed for 6 years.

Based on these phase III clinical studies, it is expected that a treatment agent for NASH will emerge in the future. Although the results of these phase III studies are pending, less than $40 \%$ of overall patients responded to the clinical trial agents. Considering that numerous molecular pathways interact to orchestrate the pathogenesis of NASH, combined therapies are likely to be utilized in the future. As each drug will likely target different aspects of pathogenesis, it is hard to predict which combined therapies will be most effective; for example, a drug targeting de novo lipogenesis combined with an anti-inflammatory agent or a drug that improves lipid metabolism paired with an anti-fibrotic agent.

It is also important to consider how genetic variations in the host influence disease phenotype. A genetic predisposition to NAFLD has been described in several studies (13). Patatin-like phospholipase domain-containing protein 3 (PNPLA3), which encodes the adiponutrin protein, identified by a genome-wide association study (GWAS), was the first gene implicated in NAFLD. 
Subsequent GWASs have verified and characterized several other variants with moderate effect sizes, including variants in GCKR, HSD17B13, TM6SF2, and MBOAT7. It is important to identify the genetic factors that regulate susceptibility to liver damage because studies in twins have indicated that heritable factors account for approximately half of the inter-individual differences in the prevalence of NASH with cirrhosis (14).

In summary, easily accessible non-invasive modalities to accurately diagnose and assess fibrosis associated with NAFLD are needed to determine treatments in clinical practice. Despite the exciting development of new pharmacological agents, it is also important to consider the value of researching new target molecules such as gut-derived serotonin (15) or combined therapies as treatment options, especially considering that many molecular pathways and numerous genetic, epigenetic, and environmental factors contribute to individual disease phenotypes.

\section{Acknowledgments}

None.

\section{Footnote}

Conflicts of Interest: The authors have no conflicts of interest to declare.

\section{References}

1. Hagström H, Nasr P, Ekstedt M, et al. Fibrosis stage but not NASH predicts mortality and time to development of severe liver disease in biopsy-proven NAFLD. J Hepatol 2017;67:1265-73.

2. Singh $S$, Allen AM, Wang $Z$, et al. Fibrosis progression in nonalcoholic fatty liver vs nonalcoholic steatohepatitis: a systematic review and meta-analysis of paired-biopsy studies. Clin Gastroenterol Hepatol 2015;13:643-54.e1-9; quiz e39-40.

3. Ekstedt M, Hagström H, Nasr P, et al. Fibrosis stage is the strongest predictor for disease-specific mortality in NAFLD after up to 33 years of follow-up. Hepatology 2015;61:1547-54.

4. Angulo P, Kleiner DE, Dam-Larsen S, et al. Liver fibrosis, but no other histologic features, is associated with longterm outcomes of patients with nonalcoholic fatty liver disease. Gastroenterology 2015;149:389-97.e10.
5. Myers RP, Elkashab M, Ma M, et al. Transient elastography for the noninvasive assessment of liver fibrosis: a multicentre Canadian study. Can J Gastroenterol 2010;24:661-70.

6. Loomba R, Wolfson T, Ang B, et al. Magnetic resonance elastography predicts advanced fibrosis in patients with nonalcoholic fatty liver disease: a prospective study. Hepatology 2014;60:1920-8.

7. Friedman SL, Neuschwander-Tetri BA, Rinella M, et al. Mechanisms of NAFLD development and therapeutic strategies. Nat Med 2018;24:908-22.

8. Yoo JJ, Kim W, Kim MY, et al. Recent research trends and updates on nonalcoholic fatty liver disease. Clin Mol Hepatol 2019;25:1-11.

9. Friedman SL, Ratziu V, Harrison SA, et al. A randomized, placebo-controlled trial of cenicriviroc for treatment of nonalcoholic steatohepatitis with fibrosis. Hepatology 2018;67:1754-67.

10. Cariou B, Hanf R, Lambert-Porcheron S, et al. Dual peroxisome proliferator-activated receptor $\alpha / \beta$ agonist GFT505 improves hepatic and peripheral insulin sensitivity in abdominally obese subjects. Diabetes care 2013;36:2923-30.

11. Ratziu V, Harrison S, Francque S, et al. GOLDEN-505 Investigator Study Group: Elafibranor, an agonist of the peroxisome proliferator-activated receptor- $\alpha$ and- $\beta$, induces resolution of nonalcoholic steatohepatitis without fibrosis worsening. Gastroenterology 2016;150:1147-1159.e5.

12. Han CY, Rho HS, Kim A, et al. FXR Inhibits Endoplasmic Reticulum Stress-Induced NLRP3 Inflammasome in Hepatocytes and Ameliorates Liver Injury. Cell Rep 2018;24:2985-99.

13. Schwimmer JB, Celedon MA, Lavine JE, et al. Heritability of nonalcoholic fatty liver disease. Gastroenterology 2009;136:1585-92.

14. Loomba R, Schork N, Chen CH, et al. Heritability of hepatic fibrosis and steatosis based on a prospective twin study. Gastroenterology 2015;149:1784-93.

15. Choi W, Namkung J, Hwang I, et al. Serotonin signals through a gut-liver axis to regulate hepatic steatosis. Nat Commun 2018;9:4824.

Cite this article as: Kim DY, Park JY. Overview of emerging treatment of non-alcoholic fatty liver disease: more than one drug needed? HepatoBiliary Surg Nutr 2019;8(5):522-524. doi: 10.21037/hbsn.2019.05.08 